\title{
Desarrollo y prospectivas de energía renovable en México
}

\section{Andrea Larios Vázquez}

En una economía dada el patrón energético queda definido por el tipo de energía utilizada de forma mayoritaria en los procesos productivos. El patrón de la economía mexicana está dominado por la energía de fuente fósil, mientras que la participación de la energía renovable en la matriz energética ha caído en lugar de incrementarse, lo que no es congruente con el gran potencial del territorio mexicano para el desarrollo de las energías solar, eólica y geotérmica, principalmente. Además, de acuerdo con los documentos de las Prospectivas de la Secretaria de Energía (SENER, 2013 c y 2013d) un posible cambio en la matriz energética requeriría la inversión del sector privado en energía eólica e inclusive nuclear, lo que cuestiona el dominio de la participación del Estado en este sector. El objetivo del artículo es analizar la evolución de la participación de las renovables en la generación de energía eléctrica, así como las diferentes perspectivas de planeación de éstas a través de los escenarios diseñados por la SENER.

La relevancia del sector energético en la economía mexicana es evidente: el crecimiento económico de las décadas pasadas estuvo impulsado por el llamado auge petrolero, y el hecho de que los ingresos petroleros son una tercera parte de los ingresos estatales. Sin embargo, las estadísticas muestran un patrón no sostenible a largo plazo, hay una tasa de crecimiento en el consumo de energía de $2.3 \%$ (promedio anual de 2003 a 2013) con una caída en la oferta bruta de energía de 1.3\% (SIE, 2014). La producción de petróleo en 2004 alcanzó su pico productivo (con 3.3 millones de barriles de petróleo al día), y según las reservas probadas totales se tendría una producción de 10 años a los niveles actuales de extracción (Gobierno de la República, 2013). Este conjunto de datos nos reafirma que en un plazo no mayor a 10 años el acceso a la energía convencional sería más complicado, lo que conlleva a la pérdida de los ingresos petroleros para el Estado. ${ }^{1}$

En el territorio nacional las renovables tienen una alta viabilidad gracias a que el país es rico en recursos naturales. La distinta distribución territorial de los recursos permite un desarrollo regionalizado: al norte la solar, al centro la geotérmica y al sur la eólica, principalmente. Según el Inventario Nacional de Energía Renovable (INER), las energías con mayor potencial son la solar y la eólica, entre

En este caso no se considera la guerra de precios emprendida los últimos meses por Arabia Saudita, que coloca el escenario en sentido inverso, al menos temporalmente, con una energía convencional abaratada al aumentar la oferta petrolera internacional. El gobierno mexicano, por ejemplo, que esperaba una gran inversión a partir de la Ronda Uno, está perplejo. 
los factores claves de su desarrollo se encuentran el desarrollo político, la reducción de costos y el desarrollo tecnológico (SENER, 2013d). La inserción de las renovables en la matriz energética se ha logrado a través de la producción de electricidad. Sin embargo, hoy en día el gas natural está cobrando relevancia debido a su bajo costo y a que es menos contaminante que otros hidrocarburos. En México su importancia ha incrementado: de 2002 a 2012 su participación pasó de 29 al 50\% en la generación de energía eléctrica, lo que contrasta con la energía de fuente renovable, que presenta una tendencia a la baja, pues de 2010 a 2013 pasó de 18 a 13.7\% (SIE, 2014).

La Gráfica 1 muestra que en 2013 la generación de electricidad se distribuyó como sigue: $82 \%$ es de fuente fósil, $13 \%$ por parte de las renovables y un 5\% nuclear. La energía hidroeléctrica es la que tiene la participación más relevante dentro de las renovables, en 2013 fue de un 10.6\% en la generación total, pero con una preocupante tendencia a la baja: en 2008 había alcanzado un $16.6 \%$. La geotérmica lo hizo con un $2.4 \%$ aunque con una ligera caída. La eólica tuvo una participación menor de $0.7 \%$, siendo

\section{Gráfica | \\ México: evolución y participación de las renovables en la generación de electricidad, 2003-2013}

-participación porcentual-

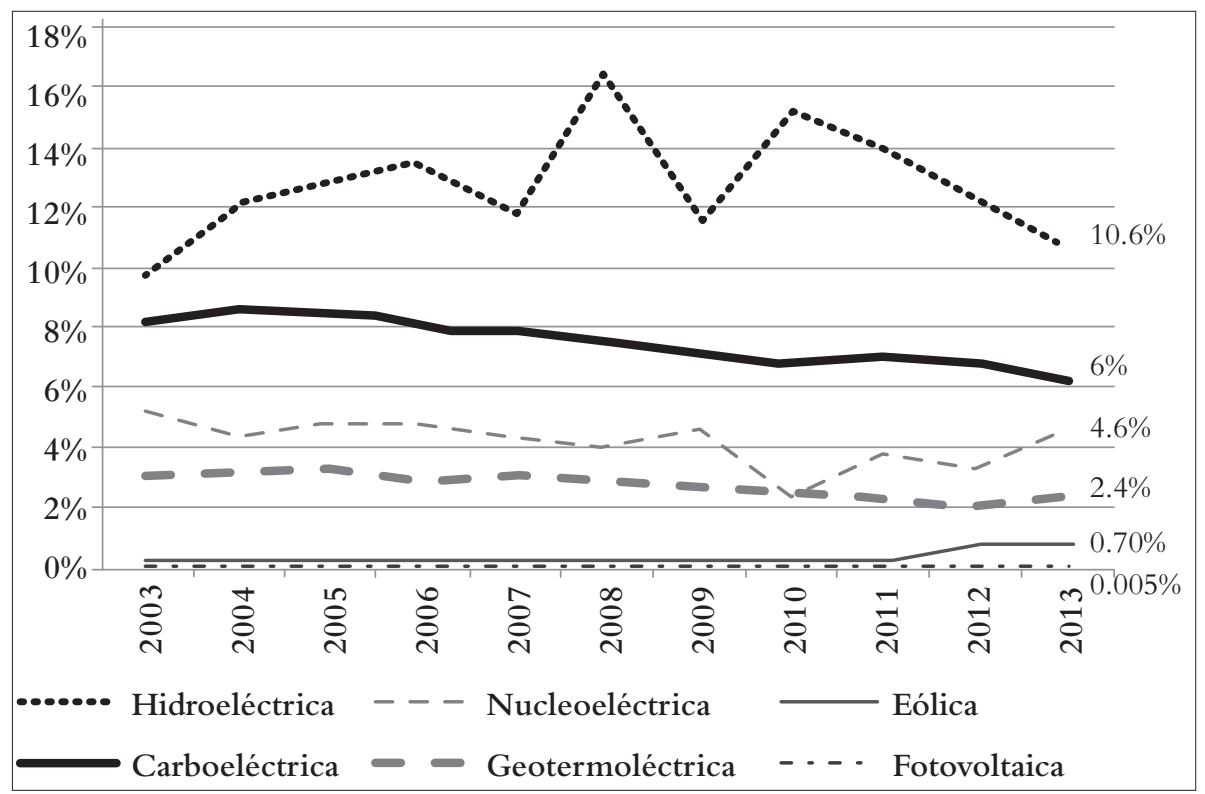

Fuente:elaboración propia con datos del Sistema de Información Energética (SIE), 2014. 
su nivel más alto en 10 años, mientras que la energía solar presentó la participación más baja, $0.05 \%$, hay que notar, sin embargo, que ésta última comenzó a utilizarse en 2012 lo que implica una tasa de crecimiento de $530 \%$ de 2012 a 2013. Mientras que la energía nuclear, por su parte, contribuyó con el 4.6\% en 2013 con una tendencia creciente.

Con el fin de incrementar la generación eléctrica con fuentes renovables, la SENER y la CFE han construido dos escenarios para su crecimiento: de "planeación” y "alterno". El primero se explica en el documento Prospectivas de Energías Renovables 2013-2027, éste estima que para 2024 la participación de la energía renovable en la generación de electricidad sea de $21 \%$, con un $2 \%$ adicional de energía nuclear y un 3\% adicional de las carboeléctricas limpias (SENER, 2OI3C). El escenario "alterno" presentado en el documento de Prospectivas del Sector Eléctrico 2013-2027 busca cumplir con las metas de la Ley para el Aprovechamiento y el Financiamiento de la Transición Energética (LAERfTE) y de la Ley General de Cambio Climático (LGCC), en particular aquélla que establece que la generación de electricidad proveniente de fuentes de energía limpia alcance al menos un 35 por ciento a partir de 2024 (SENER, 2013d). No obstante a este escenario se le puede criticar el posible incremento de la participación nuclear en la matriz energética.
En lo que respecta al primer escenario, la SENER proyecta que de 2012 a 2027 la generación total de electricidad de fuente renovable pasaría de 41,956 a 100,220Gwh/a (SENER, 2013c). Por parte de la producción del sector público dominarían las energías hidroeléctrica y eólica, mientras que la solar tendría una participación muy semejante al de la energía geotérmica. Según este escenario, el sector público y de autoabastecimiento domina en la producción de energía renovable; sin embargo, la producción de energía solar por parte de la generación distribuida es de vital importancia. En lo que respecta al segundo escenario se observa un patrón similar en la generación de electricidad: se impulsa en mayor cuantía a la energía eólica y a la nuclear, que pasan de 357 a $82,835 \mathrm{Gwh} / \mathrm{a}$ y de 10,089 a 38,879 Gwh/a, respectivamente, seguido por la hidroeléctrica que pasa de 35,796 a 43,309Gwh/a, la solar de 0 a 2,041 Gwh/a y, por último, la geotérmica de 6,507 a $8,363 \mathrm{Gwh} / \mathrm{a}$ (SENER, 2013d).

En los escenarios de "planeación" y "alterno" se impulsan en mayor cuantía a la energía eólica e inclusive a la nuclear, destacando que ésta última es no renovable. Es también de llamar la atención que las estimaciones para 2027 antes mencionadas, el crecimiento de la participación de las renovables depende del sector privado, mientras que el sector público 
proveerá en mayor cuantía energía hidroeléctrica, sin apostarle a diversificar la matriz energética, lo que con la caída en los ingresos petroleros, incrementa la incertidumbre sobre el origen de los ingresos estatales que los sustituyan. Con todo, la transición energética en nuestro país es posible y el papel del sector privado resulta fundamental para que se lleve a cabo. El deber del Estado es impulsar tal transición mediante mecanismos de regulación, competencia y fondos de inversión, además de asegurar mediante la red de transmisión que la energía renovable llegue a los consumidores de forma eficiente.

\section{Referencias}

Gobierno de la República (2013), Plan Nacional de Desarrollo 2013-2018, México.

Secretaria de Energía (SENER) (2013a), Balance Nacional de Energía 2012, México.

--- (2013b), Estrategia Nacional de Transición Energética y Aprovechamiento Sustentable de la Energía, México.

--- (2013c),Prospectiva de Energías Renovables 2013-2027, México.

--- (2013d),Prospectiva del Sector Eléctrico 2013-2027, México.

--- (2013e), Programa Sectorial de Energía 2013-2018, México, febrero.

--- (2014a), Estrategia Nacional de Energía 2014-2028, México.

--- (2014b), Inventario Nacional de Energía Renovable (INER), México, mayo.

--- (2014c), Programa Especial para el Aprovechamiento de Energías Renovables, Plan Nacional de Desarrollo 2013-2018. México.

--- (2014d),Sistema de Información Energética (SIE), México, julio. 